\title{
Small for Size Syndrome: How Small is Actually Too Small?
}

\author{
Subramaniam SRV*, Chidambaranathan S, Sathyanesan J and \\ Palaniappan $\mathbf{R}$ \\ Institute of Surgical Gastroenterology \& Liver Transplant, Govt. Stanley Medical \\ College \& Hospital, India
}

*Corresponding author: Sugi R V Subramaniam, Surgical Gastroenterology

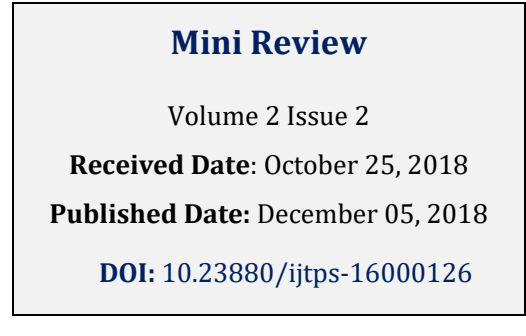

Resident, Institute of Surgical Gastroenterology \& Liver Transplant, Govt. Stanley Medical College \& Hospital Chennai, Tamil Nadu, India - 600001, Tel: +917299058581; Email: drrvsugi@gmail.com

\section{Abstract}

Kiuchi first described Small-for-size syndrome (SFSS) in recipients of small sized liver grafts with graft to recepient weight ratio (GRWR) of $<1.0 \%$ and graft volume to standard liver volume of $<35 \%$. SFSS was initially described as a spectrum of clinical manifestations resulting from the use of smaller sized liver grafts. This is more common with living donor liver transplantation (LDLT), but also has been reported with deceased donor liver transplantation (DDLT). Graft size is the only independent predictor of SFSS, however SFSS can occur even in the presence of a normal GRWR.

Keywords: Liver grafts; Hyperbilirubinemia; Liver disease; SFS grafts

Abbreviations: SFSS: Small-for-Size Syndrome; GRWR: Graft to Recipient Weight Ratio; LDLT: Living Donor Liver Transplantation; DDLT: Deceased Donor Liver Transplantation; PVP: Portal Venous Pressure; MELD: Model for End-Stage Liver Disease; PHTN: Portal Hypertension; PV: Portal Vein; HV: Hepatic Vein; MHV: Middle Hepatic Vein; IVC: Inferior Vena Cava; ECR: Estimated Congestion Ratio.

\section{Introduction}

SFSS is characterized by one or more of the following; persistent hyperbilirubinemia, coagulopathy, intractable ascites, and encephalopathy. The incidence varies from $5 \%$ to $27.5 \%$ worldwide, but can be as high as $50 \%-75 \%$ for left-lobe LDLT compared to $8.4 \%$ for right-lobe LDLT. Kyushu University defined severe SFSS as hyperbilirubinemia with a total bilirubin concentration of
$>20.0 \mathrm{mg} / \mathrm{dL}$ within a month after LDLT using left lobe grafts in the absence of technical, anatomical, immunological or hepatitis-related issues. Ikegami et al reported recipients with a model for end-stage liver disease(MELD) score of $>19$, donor age $>48$ years, and end Portal venous pressure(PVP) of $>19 \mathrm{~mm} \mathrm{Hg}$ as independent predictive factors for severe SFSS.

\section{Definitions of Small for Size [SFS]}

Definitions and criteria for SFSS have been as complicated as the management of SFSS. SFS graft was first described by Kiuchi with the use of small grafts with a GRWR of $<1.0 \%$ in LDLT. This was associated with a significantly lower first year graft survival rate of $50 \%$ to $76 \%$ compared to that of $>80 \%$ survival for middle and large-size grafts [1]. 


\section{International Journal of Transplantation \& Plastic Surgery}

Sugawara, et al. defined SFS grafts(SFSG) as those with a graft volume/standard liver volume ratio of $<40 \%$ which resulted in a significantly lower survival rate of $80 \%$ compare to that of $96 \%$ for recipients of larger grafts. Kyoto University has recently proposed that the GRWR, in combination with portal inflow modulation, can be as low as $0.6 \%$, which is probably the smallest liver graft to date. Dahm, et al. defined SFSS as a dysfunction of a partial liver graft with GRWR $<0.8 \%$, based on the presence of two of the following three criteria on 3 consecutive days during the first postoperative week, after the exclusion of other causes [2].

(1) Total bilirubin $>5.8 \mathrm{mg} / \mathrm{dL}$.

(2) Prothrombin international normalized ratio $>2$.

(3) Encephalopathy grade III.
University Hospital Zurich in 2005, came up with two different yet related terminologies, namely dysfunction and non function. SFS dysfunction was defined as dysfunctioning of a small partial liver graft with GRWR < $0.8 \%$ and SFS non function was defined as failure of a small partial liver graft with GRWR $<0.8 \%$,both during the first postoperative week after the exclusion of other causes. University of Minnesota in 2009, defined SFSS as total bilirubin $>10 \mathrm{mg} / \mathrm{dL}$ (and continuing to increase) after postoperative day 7 , coagulopathy with an international normalized ratio $>1.5$, and ascites with drain output $>2 \mathrm{~L} /$ day in the absence of an obvious technical problem such as vascular thrombosis or stenosis.

\begin{tabular}{|c|c|c|}
\hline $\begin{array}{c}\text { Recommended } \\
\text { GRWR }\end{array}$ & Study & Outcome \\
\hline $1 \%$ & Kiuchi, et al. (1999) & One year survival rate of small graft (61.2\%) vs large graft (92.6\%) \\
\hline $0.80 \%$ & Lee SD, et al. (2003) & Univariate and multiple analysis \\
\hline Less than $0.8 \%$ & Moon, et al. (2010) & One year survival rate of small graft (87.8\%) vs large graft (90.7\%) \\
\hline $0.70 \%$ & Lee SD, et al. (2014) & $\begin{array}{c}\text { Safe; no need to modulate portal pressure when using the right-lobe in } \\
\text { favorable conditions }\end{array}$ \\
\hline \multirow{2}{*}{$0.60 \%$} & Alim A, et al. (2016) & no evidence of liver steatosis in the donor graft during portal inflow \\
\cline { 2 - 3 } & & modulation \\
\hline $0.40 \%$ & Lee SD, et al. (2015) & Lowest GRWR of 0.40\% had been successfully used \\
\hline
\end{tabular}

Table 1: Various studies on determination of safe GRWR.

\section{Pathophysiology}

This phenomenon was considered to occur because of the reduced metabolic and synthetic capacity of the small graft, thereby causing delayed recovery of bilirubin clearance and prothrombin time. The loss of balance between the rapid liver regeneration and the increased demand of liver to do these functions is the principal pathogenesis of SFSS. This places the recipients at higher risk for both surgical as well as septic complications.

Portal hyperperfusion plays a central role in the development of SFSS. SFSG suffers from a transient portal hypertension (PHTN) early after reperfusion that is associated with up-regulation of endothelin-1 in the graft and ultra-structural evidence of sinusoidal damage. Consistent hyperdynamic splanchnic circulation in LDLT recipients with long-standing cirrhosis results in increased portal flow to the partial liver graft. The shear stress in hepatic microcirculation caused by elevated PVP gives rise to liver regeneration to a certain extent; however, excessive portal flow or PVP leads to a sinusoidal endothelial cell injury, which leads to SFSS and subsequent graft loss in extreme situations.

\begin{tabular}{|l|l|}
\hline \multicolumn{1}{|c|}{ Graft related factors } & \multicolumn{1}{c|}{$\begin{array}{c}\text { Recipient related } \\
\text { factors }\end{array}$} \\
\hline 1. High portal inflow & $\begin{array}{l}\text { 1. Severe preoperative } \\
\text { ESLD }\end{array}$ \\
\hline 2. Low venous outflow & 2. Poor health status \\
\hline $\begin{array}{l}\text { 3. Preexisting steatosis in } \\
\text { the donor }\end{array}$ & \\
\hline 4. Advanced donor age & \\
\hline $\begin{array}{l}\text { 5. Warm and cold ischemia } \\
\text { times }\end{array}$ & \\
\hline
\end{tabular}

Table 2: Factors that predispose to SFSS.

Decreased hepatic arterial flow caused by portal hyperperfusion occurs through a phenomenon called the hepatic arterial buffer response and has been demonstrated to contribute to ischemic biliary injury. A concomitant intestinal mucosal injury predisposes recipients to bacterial translocation, further compromising their survival. Histopathological 


\section{International Journal of Transplantation \& Plastic Surgery}

manifestations of SFSS include zonal extravasation of red blood cells into the periportal sinusoids, hepatocyte ballooning and hepatocanalicular cholestasis.

\section{Management}

Occurrence of SFSS is determined by the balance between the functional mass of the liver graft, inflow of portal vein (PV), and outflow of hepatic vein (HV) [3]. Strategies to prevent it depend upon increasing the volume of liver graft and controlling adequate PV inflow and $\mathrm{HV}$ outflow by the surgical and the nonsurgical techniques.

\begin{tabular}{|l|}
\hline 1. Final Portal venous pressure (PVP) \\
\hline $\begin{array}{l}\text { Recipients with a final portal vein pressure (PVP) } \leq 15 \\
\text { mmHg or a pressure gradient of PVP-central vein } \\
\text { pressure (CVP) } \leq 5 \mathrm{mmHg} \text { have a better prognosis. }\end{array}$ \\
\hline 2. Liver graft to spleen volume ratio \\
\hline $\begin{array}{l}\text { An early predictor of graft function in children and } \\
\text { young adults undergoing LDLT, with a ratio of }<0.88 \\
\text { predictive of portal hyperperfusion. }\end{array}$ \\
\hline 3. MELD score $>\mathbf{2 0}$ \\
\hline $\begin{array}{l}\text { 4. A decline in the platelet (PLT) count on third } \\
\text { post-operative day }>\mathbf{5 6} \%\end{array}$ \\
\hline 5. Donor age $>\mathbf{4 5}$ years \\
\hline
\end{tabular}

Table 3: Risk factors for a poor prognosis in recipients of small-for-size grafts.

\section{Increasing the Graft Volume}

Right lobe liver grafts are potentially associated with a higher incidence of morbidity and mortality in donors; therefore, vigorous attempts have been made to shift the risk from the donor to the recipient by the more liberal use of left lobe liver grafts either single or dual left lobe grafts in combination with surgical and medical interventions.

\section{Graft Outflow Reconstruction}

i. For outflow modulation; any short HV (especially from segments IV, V, VIII) larger than $0.5 \mathrm{~cm}$ are preserved and anastomosed with the recipient inferior vena cava (IVC).

ii. Middle hepatic vein (MHV) or outflow reconstruction

A small-for-size graft without MHV reconstruction can lead to various degrees of congestion of the anterior segment and a greater loss of hepatocellular function. Asakuma M, et al. established an algorithm known as the estimated congestion ratio (ECR) to decide whether MHV reconstruction is required [4]. A liver with an ECR $>0.4$ is an MHV-dominant liver, requiring MHV reconstruction or an higher GRWR grafts should be used.

$\mathrm{ECR}=$ regional volume of $\mathrm{v} 5+\mathrm{v} 8 /$ right lobe volume

\section{Graft Inflow Modulation}

In addition to outflow reconstruction, the inflow of grafts, including portal hypertension following reperfusion and the hyperdynamic splanchnic state, is reported as a major factor that can trigger SFSS. However, these views are controversial. Enhanced cholestasis, hepatocyte ballooning, disruption of the sinusoidal line, and transformation of activated Ito cells into fibroblasts are observed under the conditions of portal hypertension or overperfusion. There are different techniques for control of graft inflow which includes portosystemic shunting techniques (creation of a mesocaval shunt with downstream ligation of the superior mesenteric vein), portocaval shunts including the placement of a transjugular intrahepatic portosystemic shunt, preservation of collateral veins, splenectomy and splenic arterial ligation. Meanwhile, portal infusion of prostaglandin E1 for SFS grafts in LDLT has effectively lowered the PVP and demonstrated significantly improved liver function in the early postoperative period, suggesting that pharmacological approaches are promising in the prevention of SFSS.

\section{Pharmacological Measures}

Splanchnic vasoconstrictors, intravenous octreotides, and oral propranolol may improve the persistent hyperbilirubinemia and coagulopathy in SFSS adult recipients.

When all the surgical and pharmacological measures fail, Autologous stem cell implantation and/or a second transplant with auxiliary partial LDLT is the last resort.

\section{Conclusion}

The decision to use a liver graft can be a complex decision which requires a balance between the increasing incidences of patients dying while on waiting lists versus serious complications due to the use of a smaller/marginal graft. Graft size is the only independent and main factor for occurrence of SFSS. However, the management of this life threatening catastrophe depends upon its prevention rather than treatment. A GRWR of < $0.8 \%$ is no longer a strict critical predictor for SFSS and this can even be lowered to $0.5 \%-0.6 \%$, sometimes even 


\section{International Journal of Transplantation \& Plastic Surgery}

lower if there are accompanying positive factors of PVP $\leq$ $15 \mathrm{mmHg}$, MHV reconstruction, or young donor age. Currently, the acceptable lower threshold of the safe graft size remains at the discretion of each institution's expertise.

\section{References}

1. Kiuchi $T$, Kasahara M, Uryuhara K, Inomata Y, Uemoto S, et al. (1999) Impact of graft size mismatching on graft prognosis in liver transplantation from living donors. Transplantation 67(2): 321-327.

2. Dahm F, Georgiev P, Clavien PA (2005) Small-for-size syndrome after partial liver transplantation: definition, mechanisms of disease and clinical implications. Am J Transplant 5(11): 2605-2610.

3. Tanaka K, Yamada T (2005) Living donor liver transplantation in Japan and Kyoto University: what can we learn? J Hepatol 42(1): 25-28.

4. Asakuma M, Fujimoto $Y$, Bourquain $H$, Uryuhara $K$, Hayashi M, et al. (2007) Graft selection algorithm based on congestion volume for adult living donor liver transplantation. Am J Transplant 7(7): 17881796. 